
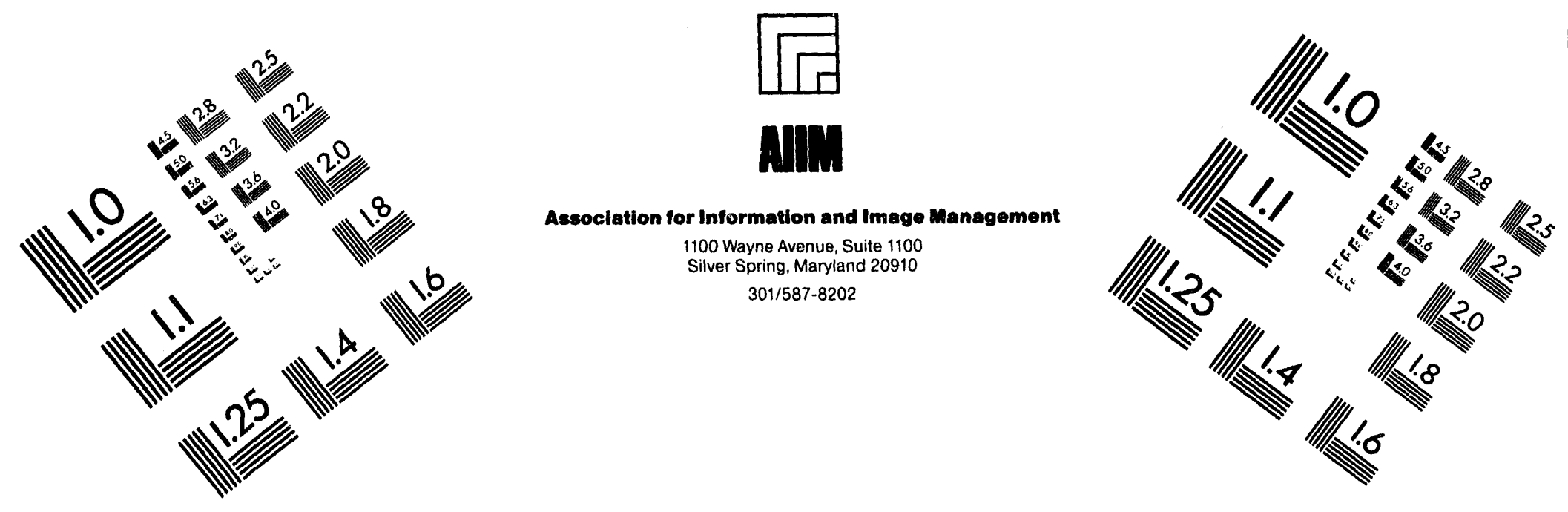

Centimeter

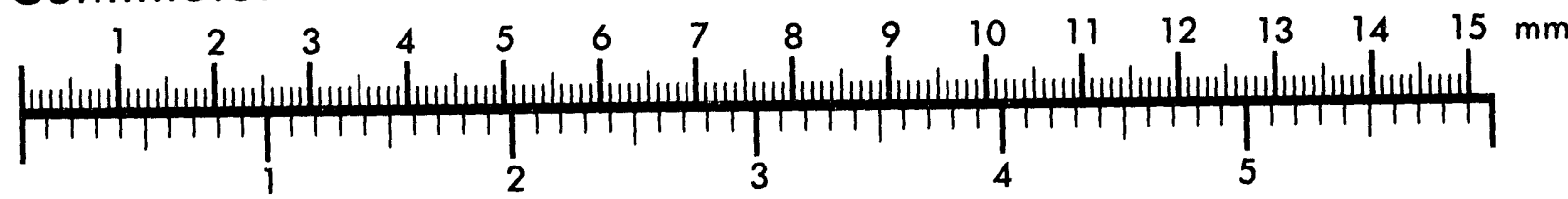

Inches
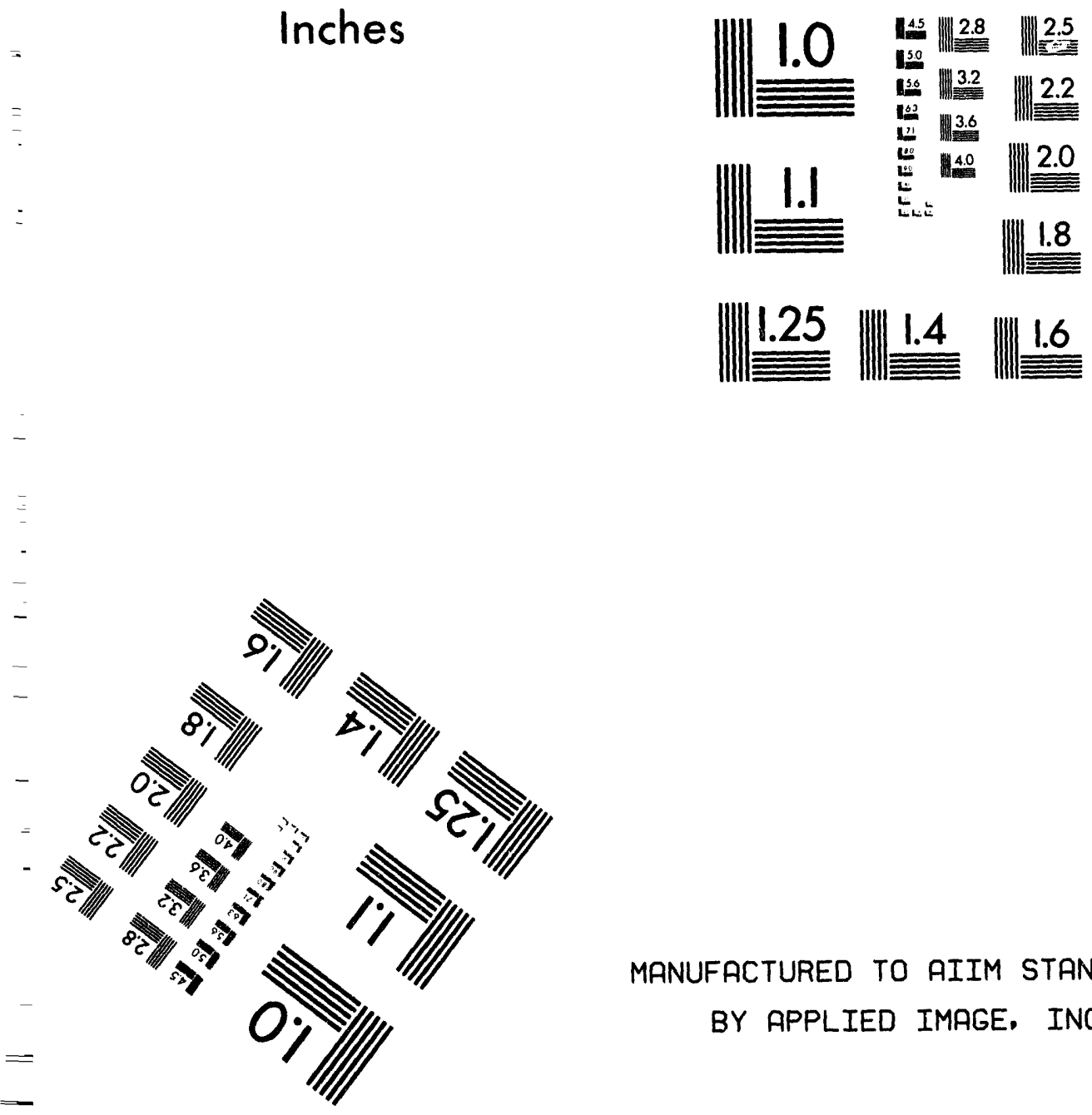

MANUFACTURED TO AIIM STANDARDS

BY APPLIED IMAGE, INC.

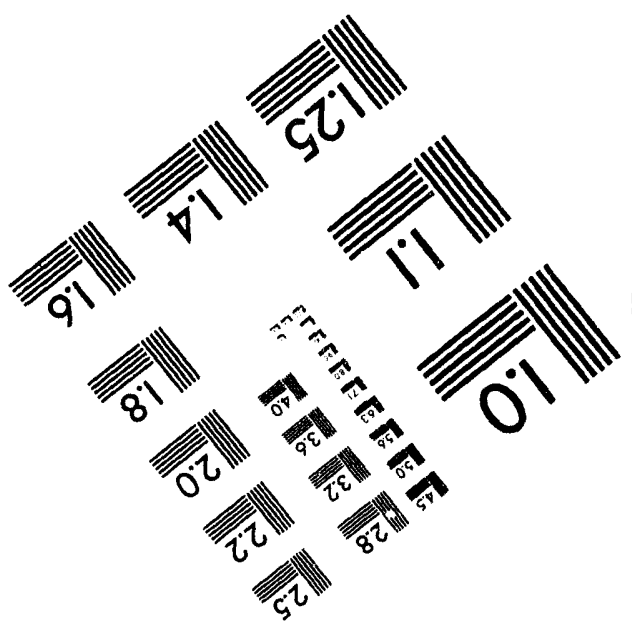



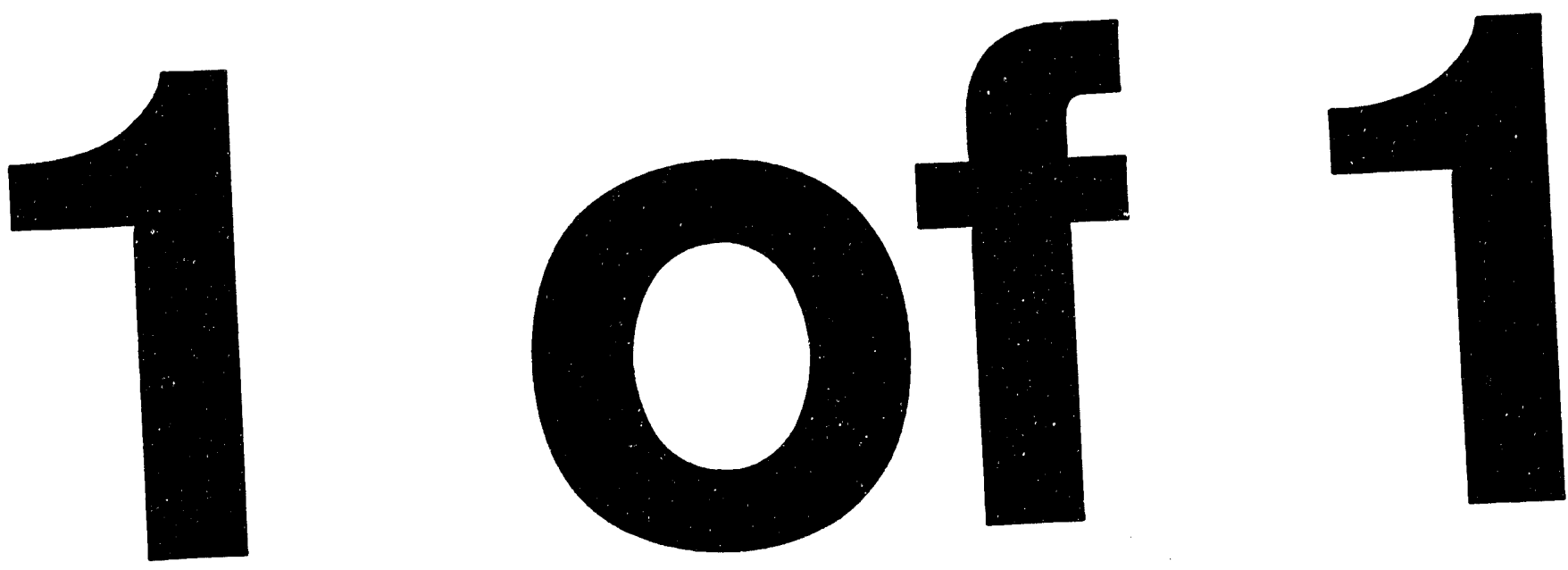

1 


\section{HSX Progress Report}

$$
\text { D) DE - ER/SZ/222-1 }
$$

May 1, 1994

Brief statements on the progress of the design and construction of the HSX experiment are reported. Topics covered include the modular and auxiliary coil systems, the coil support structure, vacuum vessel, the ECH system, the magnet power supply and site. The proposed budget for Year 2 (August 1, 1994 through July 31, 1995) is presented. The effects of a flat funding profile (based on Year 2 budget level of $\$ 1137 \mathrm{~K}$ ) on out-years and the HSX project schedule are discussed. The stretching out of the program to accommodate the reduced funding profile should result in only a slight delay in HSX operations.

\section{Main Field Coil Design}

Improvements have been made in both mechanical and magnetic design for the HSX main coil system. The coils are currently out on bid with responses due May 20, 1994. The projected final delivery schedule is two years after receipt of order. The improvements have resulted in finite-sized coils which provide a good match to the ideai single-filament spectrum, are made using relatively conventional techniques and provide increased operating margins and capabilities with respect to the power supplies.

Through improved support methods, peak stresses in the coils have now been reduced to just $\sim 46 \%$ of yield for annealed copper with a magnetic field strength of $1 \mathrm{~T}$. The safety margin in the coils, as fabricated, will be increased from this level due to the cold-working of the copper as it is laid into the coil forms. This has resulted in the elimination of the coil casings that were being considered at the time of the review (due to the stress levels present at that time).

The reduced stresses now obviate the need for the stranded strain-hardened copper/silver alloy wire used in the construction of W VII-AS. Annealed solid copper wire will be used with two directly cooled conductors incorporated into each turn of the wire pack. Heat transfer is never more that a single conductor away The copper/glass coils will be vacuum/pressure impregnated. Stress and bonding requirements can be met with proper surface preparation ( degreasing, sandblasting, priming with DZ-80 and glass overwrap) as done for JET, W VII-AS and TFTR coils. The actual epoxy system to be used is subject to testing approval after placement of order.

\section{MASTER}




\section{DISCLAIMER}

This report was prepared as an account of work sponsored by an agency of the United States Government. Neither the United States Government nor any agency thereof, nor any of their employees, makes any warranty, express or implied, or assumes any legal liability or responsibility for the accuracy, completeness, or usefulness of any information, apparatus, product, or process disclosed, or represents that its use would not infringe privately owned rights. Reference herein to any specific commercial product, process, or service by trade name, trademark, manufacturer, or otherwise does not necessarily constitute or imply its endorsement, recommendation, or favering by the United States Government or any agency thereof. The views and opinions of authors expressed herein do not necessarily state or reflect those of the United States Government or any agency thereof. 
Going to solid copper dramatically increases the packing fraction available and therefore decreases the power required to drive the coils. With the present 12 motors on site, a flat-top pulse of $150 \mathrm{~ms}$ ( at $B=1 \mathrm{~T}$ ) can be achieved with a $30 \%$ voltage headroom (at the end of the pulse) and a headroom in current-speed product of the motors of $\sim 25 \%$.

Detailed analysis of the finite-sized coil pack envisioned at the time of the review indicated an increase in the symmetry-breaking terms in the magnetic field spectrum and an increase in the rotational transform. The reason for this perturbation was the closer proximity of the inner turns of the coil to the plasma region. Increasing the spacing of the coils to plasma led to unacceptably low radii of curvature of the conductors (from larger non-planar distortions) and increased power supply requirements (due to increased coil resistance and larger stored magnetic energy). A solution was found by controlling the local winding pack orientation with respect to the centroid of the coils to better approximate the desired current path throughout the coil bundle.

The result of this procedure was obtaining a 14 turn coil pack which effectively recovers the original (design) single-filament magnetic field spectrum and rotational transform profile. Coil feed locations, cross-overs from pancake-to pancake and conductor transitions (jogs) from turn-to-turn in this region have all been modeled. The feed locations are influenced by coil support structure interferences and the impact on the magnetic field structure. Calculations with realistic feeds and cross-overs show minimal effects on the flux surfaces and helically symmetric structure of the fields with the design adopted.

\section{Coil Support}

The key to eliminating the coil casings was providing adequate support for the coils. Both radial loads ('hoop' forces) and lateral loads (transverse to the coil large cross-section dimension) are significant. Finite element analysis showed the need for coil support on the outboard side of the device.

The solution is a set of planar stainless steel rings which surround each coil. The rings are $3 / 4$ of an inch thick ('toroidal direction') with a $10 \mathrm{~cm}$ radial thickness. The rings provide significant stiffness in the radial direction and are attached to the coils with brackets at the needed support locations. The brackets are shimmed to the coils with epoxy after transfer of alignment points from the coils to the rings. With the exception of the alignment points, these are non-precision components. 
The lateral loads are taken up by coil 'rigs', which transmit the lateral loads from coil-to-coil around the torus effecting cancellation. The lateral loads are thus taken in compression with epoxy shims from the actual rigs to the coil surface proper. The rigs are supported off of the coil rings. HSX can operate at low field $(2 \mathrm{kG})$ without the rigs. Initially, field mapping will be performed at $2 \mathrm{kG}$ to confirm the proper position of all the coils. After confirmation, the epoxy shims between the coils and rigs will be installed to 'lock' the structure into the operating configuration.

The rings are supported off of a central support structure which takes up the predominate centering force and the torques present within a field period and serves to align the individual field periods. A preliminary design consisting of pipes and cones and positioning/supporting elements has been proposed. Current investigations are attempting to simplify this structure and reduce the cost.

\section{$\underline{\text { Auxiliary Coils }}$}

An inexpensive auxiliary coil set has been designed which permits small changes in the rotational transform profile (without large detrimental effects on the spectrum), can be used to add strong symmetry-breaking terms to the spectrum for the physics program, and can introduce a deep (2\%) magnetic well into the configuration.

The planar coil support rings offer a convenient and inexpensive method to incorporate the flexibility provided by a set of trim coils. The preliminary design of these coils calls for a 10 turn double-pancake coil to be incorporated onto each ring. The coil follows the shape of and is centered on the ring allowing an inch on the inside of the ring for coil/rig attachment and an inch on the outside of the ring for attachment to the support structure. The coils are therefore planar and have no negative curvature so they can be made with conventional fabrication techniques (winding under tension). At nominal field they can be driven with a single motor/generator without adversely impacting operation of the main field set ( especially if we install the remaining four M/G's from Los Alamos - see below).

These coils can provide modest rotational transform control without seriously degrading the quasi-helical symmetry. With $4 \%$ of the main coil MMF the auxiliary coils can produce a $3.6 \%$ increase in the rotational transform. With $9.3 \%$ of the main $\mathrm{MMF}$, the rotational transform can be increased to a resonance-free region from 1.15 to 1.24 with a magnetic well of $2 \%$. 
By energizing a select subset of these coils at $10 \%$ of the main coil current, the toroidal mirror term in the spectrum can be increased to levels $\left(8.5 \% \mathrm{~B}_{\mathrm{O}}\right)$ where neoclassical transport can be significantly increased ( at least an order of magnitude ) in the center of the device. From measurement of the electron temperature profile one can determine whether the quasi-helical symmetry has a beneficial effect on the electron thermal conductivity.

This location of the trim coils does not result in increased difficulties for diagnostic access. The main coils, support rings and trim coils form a compact modular unit for incorporation into the whole device.

\section{Vacuum Vessel}

The vacuum vessel for HSX is completely independent of the magnet set and is not required to support any magnetic loads ( other than eddy currents). The vessel shape has been designed to provide approximately $3 \mathrm{~cm}$ clearance between the wall and plasma. The vessel is assembled into field period units with a flanged connection in the 'straight' sections of the torus. The flange and vessel sections are such to permit the installation of the coils after the main vacuum vessel joints and sections have been completely assembled and tested.

The vessel will be made from $1 / 4$ " type 316 stainless steel. A $5 \mathrm{~mm}$ (.2") wall was found adequate to support the vacuum, gravitational and eddy current loads using a finite element analysis. The analysis showed a maximum wall deflection under load of less than $.5 \mathrm{~mm}$ and equivalent stresses in the steel of under $40 \mathrm{MPa}$ ( yield point $\sim 240 \mathrm{MPa}$ ). Given the maximum possible error as calculated by ANSYS, the safety factor is reduced from 6 to only 4 . Ports and flanges have not been incorporated into the model, but are predicted to increase this safety factor due to the increase in cross-section. The weight of each field period is roughly 500 pounds.

Two methods have been explored for fabrication: bending of plane developments and explosive forming. Test pieces using plane developments showed difficulties in holding tolerance for joining the smaller sections into a torus. Explosive forming has been selected as the method of choice. Tests were made forming a $3 / 8^{\prime \prime}$ thick steel tube into the vessel section with large ellipticity and indentation ('beany' cross-section). A concrete mold was used for this test. The results showed the steel could be moved into good conformation with the mold shape after explosion. Cross-section measurements showed only a $6 \%$ variation in thickness after forming. 
The vessel will be made in 16 segments, each $1 / 4$ of a field period in length. The mold is defined by a cylindrical tube which encircles and is coaxial with an entire $1 / 2$ period of the torus (the other half is identical). Flat disk sections with an OD equal to the ID of the cylinder have the exterior shape of the vessel machined in the appropriate positions. Also incorporated into these mold rings are marks to define locations on the vessel and parting lines for subsequent joining. To simplify the preforms and reduce the length of the mold-ring confining cylinder, the $1 / 2$ period vessel sections are formed out of two pieces. These are subsequently joined by welding using the mold rings as clamping and aligning fixtures. The period joining flanges are accurately positioned in a fixture and welded to the tube. Any misalignments are corrected at the 'box ports' (see below) at the 'corners' of the vacuum torus.

Because the coils can be slipped over the torus sections into their final positions, the vessel can be preassembled and leak-checked with the period joints and box ports installed. After confirmation of geometry and vacuum integrity, the holes for other ports will be cut into the vessel and welded after installation of the coils.

The explosion forming mold design and drawings are complete and fabrication will commence in the near future. Goals are to have a proto-type section completed in August of this year.

\section{Diagnostics and Porting}

The primary aim of the HSX is to determine whether the reduced neoclassical transport of the quasi-helical stellarator compared to a conventional stellarator has an observable effect on the global and local particle and energy confinement. Auxiliary coils will provide a means by which the magnetic field spectrum can be altered and the neoclassical losses greatly enhanced. The primary aim of the initial diagnostic set is to determine the power balance during electron cyclotron heating. This will enable us to ascertain whether the experimental electron thermal conductivity has been noticeably affected by the change in the magnetic field spectrum. We anticipate that with comparatively low density ECH plasmas the ion losses will be dominated by charge exchange and not by neoclassical transport and that the coupling between the ions and electrons will be small. In allocating space on the vacuum vessel for ports, we have provided for the future upgrading of the diagnostic set to include HIBP for electric field measurements, FIR for full density profile information, reflectometer for density fluctuation measurements and duo-spectrometers for impurity rotation. 
All HSX porting can be divided into two types, boxports and portholes. There will be four boxports located at the four corners of HSX and a number of portholes of different sizes for probe work and feed-through of the internal diagnostics.

A number of diagnostics will be located on boxports that provide the maximum access to the plasma. The boxports are being designed to accommodate a variety of diagnostics fairly easily. The Thomson scattering system that was formerly used on IMS will provide density and temperature data at 8 radial locations. Also, a 2 -chord $70 \mathrm{GHz}$ microwave interferometer will provide line-average density measurements. For neutral density profiles we will have an array of 8 chords of H-alpha detectors. A $1-\mathrm{m}$ monochrometer will be used to measure the blue and red shift of selected impurity lines for flow velocity measurements. Soft $X$-ray detectors will provide energy spectra and profile information of high energy electrons during the ECH.

The boxports are designed to accomplish four tasks. First, they can accommodate the diagnostics that can provide multichordal information and require large access to the plasma such as HIBP, X-ray tomography, microwave and FIR interferometry, Thomson scattering, and $\mathrm{H}$-alpha monitors. Second, they can be used to absorb unexpected misalignments that occur when the two half-periods of HSX are assembled. Third, they are designed to have a large conductance for extra vacuum pumping of the HSX vessel, if necessary, without reducing diagnostic access. Fourth, the vacuum vessel can be supported from the four boxport locations.

The vacuum vessel of HSX will be pumped through two large ports that are spaced 180 degrees apart toroidally. According to the expected outgassing and conductance of the ports, each of the two identical vacuum systems will have an effective pumping speed of about $700 \mathrm{l} / \mathrm{s}$. Base pressures on the order of 10-8 Torr are expected with moderate cleaning of the vacuum vessel surface. Additional pumping speed, with a higher conductance, can be obtained through use of tanks mounted on the box ports if necessary.

\section{$\underline{\mathrm{ECH}}$}

Discussions are near completion with LLNL on arrangements to transfer the TMX-U gyrotron power supplies to Wisconsin. Available materials include the supplies themselves, the high voltage cables to the gyrotrons, the gyrotron magnet power supplies, the remote control chassis for the gyrotrons and a large amount of waveguide and components. Notably missing are the controller for the magnet power supplies and the FC-75 window cooling 
system for the tube. All schematics for missing electronic components have been identified and located and will be provided with the transfer. Additionally, Carl Williams, who was the lead engineer for the TMX-U ECH has agreed to assist us in the disassembly and reassembly of the supplies at Wisconsin. Preliminary arrangements are to disassemble the supply this July with subsequent shipment to Wisconsin. Reassembly will occur after the HSX site is ready. Reassembly is presently scheduled for May 1995.

The tubes, socket tanks and controls for the gyrotron are at Oak Ridge. A visit to ORNL to inspect these components and begin discussions on arranging the transfer is scheduled for the near future. At the same time, the ATF staff will be bringing up a $28 \mathrm{GHz}$ system for long-pulse ( $>1$ hour) operation on ATF. HSX personnel will help with the initial set-up and operation to gain experience.

An initial offer from the Garching Wendelstein VII-AS group to provide some of the compcients from their $70 \mathrm{GHz}$ WVII-AS-AS ECH system is being pursued. This system includes the FC-75 cooling system, the $70 \mathrm{GHz}$ long pulse tube and magnet, all associated controls and modulators, arc detectors, k-spectrometers, transmission lines, torus window, and other components which will be useful in setting up the HSX system.

\section{Magnet Power Supply}

Charge and discharge tests have been performed on three of the MG units with encouraging results. The drive supplies have been sized from experimental data. With the present 12 motors on site, a flat-top pulse of 150 ms ( at $B=1 \mathrm{~T}$ ) can be achieved with a $30 \%$ voltage headroom (at the end of the pulse) and a headroom in current-speed product of the motors of $\sim 25 \%$.

Initial tests have been performed on a set of the motor/generators to provide data on their operating characteristics for sizing the drive supplies. To date three of these units have been tested. The grease, brushes and commutators are all in good shape. Minor repairs to the insulation on the leads and cleaning of the commutators has been necessary. These units have been run at up to 2200 RPM (10\% past peak operating speed). They all seem to improve (in reduced current draw at speed) with increasing use. Tests have been performed to determine the friction and windage losses as well as the fieldarmature coupling coefficient. The results on coupling are similar to those obtained in testing at LANL (friction and windage results were not available).

The results show that an armature supply of 20A at $600 \mathrm{VDC}$ is capable of bringing a motor/flywheel assembly up to speed from 1600 to 2000 RPM within the required 5 minute machine cycle. To allow for an additional 4 $\mathrm{M} / \mathrm{G}$ units, the armature drive has been sized to $320 \mathrm{~A}$. With the measured 
values of coupling coefficient, the field drives have been sized. Again, allowing for the possibility of 16 units the field supply must be intermittently capable of $300 \mathrm{~V}$ at $1000 \mathrm{~A}$ to accommodate flat-topping of the current waveform. The supply is rated $\mathrm{DC}$ at $1000 \mathrm{~A} 100 \mathrm{~V}$ to provide current for the HSX main field for ECH discharge cleaning at $870 \mathrm{G}$ ( $2.45 \mathrm{GHz}$ resonance).

Initial discharge tests have been performed into a dummy load. Because of limitations on the presently available field supply, these tests have been limited to full design current, but at lower voltages. Discharges have been performed at $1100 \mathrm{~A}$ at a speed of $800 \mathrm{RPM}$ for an I $\omega$ product of .88 megaampere-RPM. This should be compared to a value of .5 needed for operation at $.5 \mathrm{~T}$ (for second harmonic at $28 \mathrm{GHz}$ ) and 1.8 for full design value. Subsequent tests at higher levels are planned for the near future. A dual system of speed monitors, one optical and the other a mechanical tachometer, is under design and test.

Four additional motors have been promised to us by LANL, if they are needed. Because of the additional voltage/current headroom resulting from the improved coil design, our philosophy is to size the critical elements to accommodate the extra units if needed, b't not to install the extra four motors initially. With these additional mo rs, a $250 \mathrm{~ms}$ pulse can still be attained even with a $25 \%$ increase in the coil resistance. With 16 motors and $\mathrm{B}=1.1 \mathrm{~T}$, the voltage headroom is over $50 \%$ and the current-speed headroom is about $57 \%$.

With 16 motors, the coil set would be capable of operation at $1.25 \mathrm{~T}$ with a flattop of $100 \mathrm{~ms}, 15 \%$ voltage and $30 \%$ current-speed headroom. Stresses in the coils would then be running at $\sim 72 \%$ of yield for annealed copper. This scenario would allow operation of HSX at some time in the future using 70 $\mathrm{GHz}$ gyrotrons for second-harmonic heating. The Garching group has indicated that the W VII-AS systems could be made available, as mentioned earlier, for this effort as they are going to $140 \mathrm{GHz}$ Russian systems.

The motor testing and refurbisining will be completed by November 1994 . After each unit is checked out, it will be moved to indoor storage to await installation in the pit. Installation is presently planned for February 1995 if the site modification permits.

Site

The approval process for the site preparation is going forward at a good pace. The proposal has been approved by both the Board of Regents and the State Building Commission. Within the next 6-8 weeks an Architect and Engineering firm will be appointed for the final design. Best estimates are for the bidding process to be completed in August or September 1994 . Construction completion is hoped for by February 1995. 

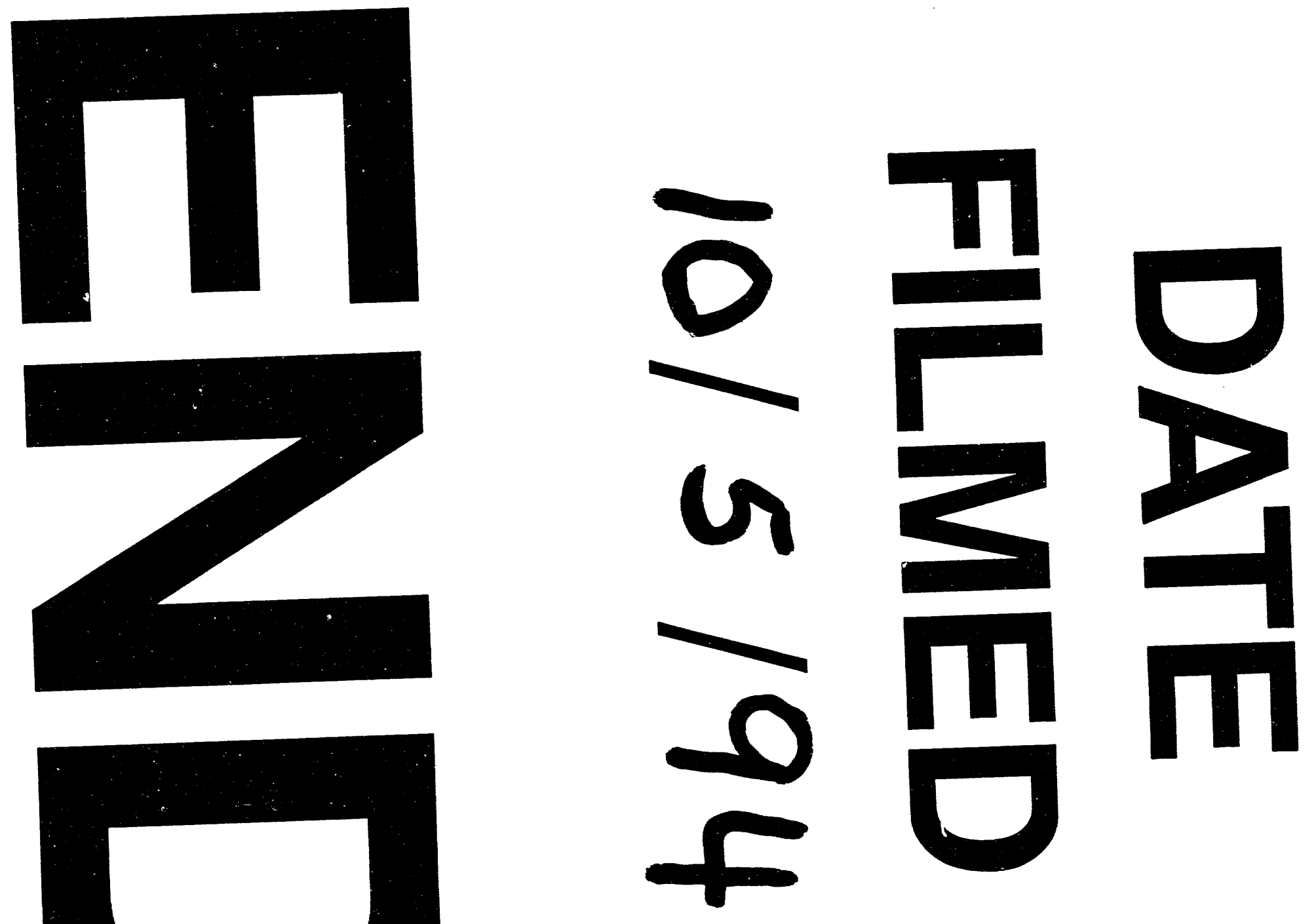
